\title{
Erfolgreicher Tabubruch
}

\section{Adrian Ritter}

Freier Journalist

\author{
Im Mai 2014 gelang es den Haus- und Kinderärzten in einer Volksabstimmung, die \\ medizinische Grundversorgung in der Bundesverfassung zu verankern. Der Weg \\ zu diesem Ziel war auch ein Aufbruch zu mehr Selbstbewusstsein. Die Publikation \\ Mut zur Wut blickt auf die bewegte Zeit zurück.
}

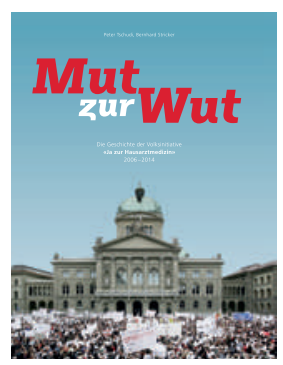

Peter Tschudi, Bernhard Stricker Mut zur Wut

Die Geschichte der Volksinitiative «Ja zur Hausarztmedizin» 2006-2014 Basel: EMH Schweizerischer Ärzteverlag; 2015.

480 Seiten, $25 \mathrm{CHF}$.

ISBN

978-3-03754-088-6
Es war kein Aprilscherz. Die Hausärzte und Kinderärzte meinten es ernst am 1. April 2006. Rund 12000 Ärztinnen und Ärzte sowie unterstützende Personen demonstrierten auf dem Bundesplatz in Bern für eine Stärkung der Hausarztmedizin. Es war ein Tabubruch: Nie zuvor hatten Ärzte auf der Strasse für ihre Anliegen demonstriert. Das Fass zum Überlaufen gebracht hatte der Entscheid des damaligen Gesundheitsministers Pascal Couchepin, den Labortaxpunktwert um zehn Prozent zu kürzen. Aufgestaut hatte sich ein grundsätzlicher Ärger über die politische Vernachlässigung der Hausarztmedizin. Die Hauptbotschaft der Demonstrierenden auf dem Bundesplatz: Ohne Massnahmen zugunsten der Hausarztmedizin droht der Schweiz ein gravierender Hausärztemangel.

Die Kundgebung in Bern verlieh nachhaltigen Schub. In der Folge lancierten die Haus- und Kinderärzte die Volksinitiative «Ja zur Hausarztmedizin». Gleichzeitig wich mit Didier Burkhalter und später Alain Berset als Gesundheitsministern die Konfrontation zwischen Bund und Hausärzteschaft zunehmend einer partnerschaftlichen Zusammenarbeit. In einem Masterplan griff der Bundesrat wichtige Anliegen der Volksinitiative auf etwa den Ausbau der universitären Hausarztmedizin, die Förderung von Praxisassistenzstellen und eine Besserstellung der Hausärzte in der TARMED-Tarifstruktur. Dies führte dazu, dass die Initianten das Volksbegehren 2013 zugunsten eines direkten Gegenvorschlages zurückzogen. Dieser kam im Mai 2014 zur Abstimmung und erzielte das fünftbeste Resultat aller Volksabstimmungen in der Schweiz: 88,1 Prozent Zustimmung. Die soeben erschienene Publikation Mut zur Wut zeichnet die Erfolgsgeschichte von der Demonstration bis zur Volksabstimmung minutiös nach. Herausgegeben haben das Werk zwei wichtige Akteure der Geschehnisse - der Basler Allgemeinmediziner Peter Tschudi und der Journalist Bernhard Stricker. Peter Tschudi war Gründer und Vorsteher des schweizweit ersten Instituts für Hausarztmedizin an der Universität Basel. Bernhard Stricker war früher Medienbeauftragter der Schweizerischen Gesellschaft für Allgemeinmedizin
(SGAM), hatte die Idee zur Volksinitiative und war später deren Medienbeauftragter.

Mut zur Wut leistet einen Beitrag zu Schweizer Geschichte und Politik und lässt eine für die Ärzteschaft prägende Zeit nicht in Vergessenheit geraten. Das attraktiv aufgemachte und bebilderte Buch beschreibt, wie es der Ärzteschaft erstmals gelang, Patienten als politische Verbündete zu gewinnen mit dem gemeinsamen Ziel, eine starke Hausarztmedizin aufrechtzuerhalten. Tschudi und Stricker sind überzeugt, dass der Erfolg nicht zuletzt der Glaubwürdigkeit der Hausärzte zu verdanken ist und dass es klug war, sich mit dem Anliegen nicht an eine politische Partei zu binden. Das Initiativkomitee bestand ausschliesslich aus Hausärztinnen und Hausärzten.

Was die Schweizer Haus- und Kinderärzte zwischen 2006 und 2014 geleistet haben, ist politisch einzigartig, sind die Herausgeber des Buches überzeugt: Sie haben nicht nur die medizinische Grundversorgung in der Bundesverfassung verankert, sondern die Hausärzte endlich zu gleichwertigen Partnern im Gesundheitswesen werden lassen. Das Buch lässt Personen zu Wort kommen, die einen wichtigen Beitrag zum Erfolg geleistet haben, Entscheidungsträger aus dem Gesundheitswesen und - als einziger Kritiker der Volksinitiative, der sich als Autor zur Verfügung stellte SVP-Nationalrat Toni Bortoluzzi. Er prophezeit, dass sich der Ruf der Hausärzte nach staatlicher Unterstützung früher oder später gegen sie richten werde im Sinne einer Verstaatlichung des Gesundheitswesens. Die Autorinnen und Autoren von Mut zur Wut haben anderes im Sinne. Sie wollen die Verantwortung für ihre neue starke Rolle in der Gesundheitspolitik wahrnehmen - etwa im Rahmen des neuen Berufsverbandes «Hausärzte Schweiz» (MFE). Nach "gewonnener Schlacht» dürfe man nicht die Hände in den Schoss legen, fordert MFE-Präsident Marc Müller. Es gelte, den Elan zu nutzen: Die medizinische Grundversorgung müsse neu gedacht werden und ein Dialog über die Kernkompetenzen der verschiedenen Berufsgruppen stattfinden. 